\title{
Simple, readily available clinical indices predict early and late mortality among patients with ANCA-associated vasculitis
}

\author{
Ágnes Haris ${ }^{1}$, Kálmán Polner ${ }^{1}$, József Arányi ${ }^{1}$, Henrik Braunitzer ${ }^{1}$, llona Kaszás ${ }^{2}$, László Rosivall ${ }^{3}$, Gábor Kökény ${ }^{3 *}$ \\ and István Mucsi ${ }^{4}$
}

\begin{abstract}
Background: The early identification of patients with ANCA-associated vasculitis (AAV) who are at increased risk for inferior clinical outcome at the time of diagnosis might help to optimize the immunosuppressive therapy. In this study we wanted to determine the predictive value of simple clinical characteristics, which may be applicable for early risk-stratification of patients with AAV.

Methods: We retrospectively analyzed the outcome of 101 consecutive patients with AAV receiving a protocolized immunosuppressive therapy. Baseline Birmingham Vasculitis Activity Score (BVAS) and non-vasculitic comorbidities were computed, then predictors of early ( $<90$ days) and late ( $>90$ days) mortality, infectious death, relapse and end stage kidney disease (ESKD) were evaluated.

Results: The baseline comorbidity score independently predicted early mortality (HR 1.622, Cl 1.006-2.614), and showed association with infectious mortality (HR 2.056, CI 1.247-3.392). Patients with BVAS at or above median $(=21)$ had worse early mortality in univariable analysis ( $\mathrm{HR} 3.57, \mathrm{Cl} 1.039-12.243)(p=0.031)$, and had more frequent relapses $(p=0.01)$ compared to patients with BVAS below median.

Conclusions: Assessing baseline comorbidities, beside clinical indices characterizing the severity and extension of $A A V$, might help clinicians in risk-stratification of patients. Future prospective studies are needed to investigate whether therapies based on risk-stratification could improve both short term and long term survival.
\end{abstract}

Keywords: ANCA, BVAS, Comorbidity, Immunosuppression, Outcome, Vasculitis

\section{Background}

The outcome of ANCA-associated vasculitis (AAV) has improved significantly since the introduction of immunosuppressive therapy. On the other hand, both the disease and the cytotoxic treatment are associated with considerable morbidity and mortality $[1,2]$. Ideally, patients should receive a treatment specifically tailored to the severity of their disease. Other factors, however, such as age, the extent of organ involvement and also baseline comorbidities may influence the outcome [3-7]. Therefore, in order to optimize the intensity of immunosuppression and to optimize outcomes, these factors would be important to

\footnotetext{
*Correspondence: kokeny.gabor@med.semmelweis-univ.hu

${ }^{3}$ Institute of Pathophysiology, Semmelweis University, 4 Nagyvárad tér,

Budapest 1089, Hungary

Full list of author information is available at the end of the article
}

consider when planning the treatment schedule of the individual patient at the time of diagnosis.

The Birmingham Vasculitis Activity Score (BVAS) is a reliable tool to estimate the severity and extent of the disease [8]. However, studies investigating it's predictive value on survival reported conflicting results [9-13]. Beside the activity of AAV, patients may have comorbid conditions, that may also have an impact on their survival. Comorbidity scores are useful clinical tools for risk-stratification of patients with chronic disorders, and the role of comorbid conditions has been emphasized in the mortality of dialysis patients [14, 15]. Therefore, it seems reasonable to include comorbidity assessment in the initial risk-startification at the time of presentation with AAV. 
The aim of our study was to determine if simple clinical and laboratory characteristics, readily available at the time of diagnosis would predict mortality in patients with BVAS. We assessed BVAS, and utilized a simplified score by computing the most important baseline nonvasculitic comorbidities for risk-stratification of patients with AAV.

\section{Methods}

All consecutive patients, diagnosed with AAV at our nephrology center between January, 1998 and June, 2013 were considered for this study. One patient, who died within the first month, and 3, who were lost to followup were excluded. Last follow-up was the date of death or the end of study (December 31, 2013). Patients who survived longer than the 8 years of follow-up $(n=15)$ were censored at that time.

The diagnosis of necrotizing small vessel vasculitis was defined according to the criteria of Chapel Hill consensus conference [16, 17], by clinical presentation compatible with AAV, positive ANCA serology and/or kidney biopsy. Histological result confirmed the presence of crescentic/ necrotizing glomerulonephritis in all but 10 subjects, in whom renal biopsy was not performed either because of life threatening condition or due to refusal by the patients. All these 10 patients were ANCA positive. Estimated GFR (eGFR) was computed with CKD-EPI equation [18], and BVAS (version 3) was calculated by scoring symptoms in 9 organ systems (general, cutaneous, mucus membranes/ eyes, ENT, chest, cardiovascular, abdominal, renal, and nervous system) at admission [8] (Evaluelogix software by EPS Research Ltd). Baseline comorbidity score was assessed by determining conditions that had been present before the AAV, namely history of myocardial infarction, congestive heart failure, peripheral vascular disease, cerebrovascular disease, chronic pulmonary disease, peptic ulcer disease, liver disease, diabetes or malignancy. Scores were given 0 if no comorbidity, 1 if a single comorbidity, 2 if two or more comorbidities existed.

Patients received protocolized therapy during the entire observational period: 500-1000 mg intravenous (iv) metlyprednisolone (MP) for three consecutive days, followed by $1 \mathrm{mg} / \mathrm{kg} /$ day per os for one month, then daily $48 \mathrm{mg}$ in the second, $36 \mathrm{mg}$ in the third, $24 \mathrm{mg}$ in the fourth, 16 then $12 \mathrm{mg}$ in the fifth, and 8 then $4 \mathrm{mg}$ in the sixth months, continued with the maintenance dose of $4 \mathrm{mg} / \mathrm{day}$, and $10 \mathrm{mg} / \mathrm{kg}$ iv bolus cyclophosphamide monthly for six months, repeated at months 9 and 12. For subjects older than 65 years the dose of immunosuppressive medications was decreased by $15 \%$, and for older than 70 years by $20 \%$, but the CYC dose was not modified by the glomerular filtration rate. In 92 patients five plasmapheresis sessions were also performed. Eighty-six patients followed the protocol strictly. When we analyzed their data separately, the results were comparable to the findings in the whole cohort. After twelve months azathioprine was introduced, accompanied by $4 \mathrm{mg}$ methylprednisolone given daily or every other day as long-term maintenance therapy, at the discretion of the attending nephrologist.

In case of relapse, the induction immunosuppressive regime was repeated.

Remission was defined as disappearance of clinical disease activity and stabilization or improvement of the kidney function. Resolution of hematuria was also criteria for remission, but persistent proteinuria was considered as the consequence of glomerular damage. In patients who remained dialysis dependent we considered remission if the extrarenal manifestations and the hematuria completely ceased. Relapse was defined as recurrence of presenting symptoms or appearance of a new organ involvement attributable to AAV. Those, in whom remission could not be achieved, who died due to active vasculitis, or had low grade of persistent "grumbling disease" were considered as treatment resistant patients.

The main exposure variables were the comorbidity score (the sum of comorbidities at the time of admission) and the BVAS score (categorized as below or above median $[$ median $=21]$ score).

The primary end points were all cause early ( $<90$ days) and late ( $>90$ days) mortality. Secondary end points consisted of deaths due to infections, rate of relapse and end stage kidney disease (ESKD).

Statistical analyses were performed using SPSS 20.0 (IBM, Chicago, IL) and STATA MP version 12 (Stata Corporation, College Station, TX). Variables were reported as mean (SD) or median and range, comparison between groups was analyzed by Student's t-tests, Mann-Whitney $U$ tests or $\chi^{2}$ tests, as appropriate. Mortality risk was calculated by Kaplan-Meier method, and log-rank tests to compare groups. Predictors of death were evaluated separately for early ( $<90$ days $)$ and late (>90 days) mortality.

Patients with BVAS below and at or above median were compared. Although the relatively small number of events limited multivariable analyses [19], for this purpose those variables were selected, that were considered important predictors of outcomes of AAV based on clinical experience or the results of the univariable analyses.

Multivariable models were sequentially adjusted for age, serum albumin, HD dependency on admission, and ANCA type (negative, p- or c-ANCA). Serum CRP was not used in the multivariable models due to the small number of events and also because of its strong correlation with serum albumin.

Logistic regression models were used to analyze the association between exposure variables and relapse, since we considered all relapses for these analyses and we did not consider the time to events. 
Results are expressed as hazard ratios (HRs) with 95\% confidence intervals (CIs) and p values. All tests were two-tailed, unadjusted for multiple comparisons, and $p$ values of $<0.05$ were considered significant.

\section{Results}

Baseline data of the 101 individuals are presented in Table 1. Subjects with BVAS at or above median (median BVAS $=21)$ had lower Hgb $(p=0.017)$, more c-ANCA positivity $(p=0.024)$, and needed HD on admission more often $(p=0.012)$, compared to the individuals with BVAS below median.

Treatment protocol was strictly followed with only few exceptions, as excluded iv MP pulses in 1 and 2 patients and excluded CYC boluses in 3 and 1 patients in the BVAS at or above and below median groups, respectively; CYC was administered orally in 2 patients in the BVAS below median group. Subjects with BVAS at or above median got higher dose of pulse MP $(p=0.002)$ compared to the individuals with BVAS below median, but the dose of CYC and the cumulative dose of MP did not differ between the groups (Table 1).

The median survival in the study sample was 1877 (95\%CI 753-2246) days. Mortality during the first year was $33 \%$. Nineteen patients died within the first 90 days ("early mortality"), and 41 after the $90^{\text {th }}$ day of follow-up ("late mortality"). The cumulative probability of survival was 0.441 (95\%CI $0.231-0.633)$ versus 0.233 (95\%CI $0.126-0.359)(p=0.028)$ in patients with a BVAS score below versus at or above median, respectively (Fig. 1). The cumulative probability of early (within 90 days after diagnosis) survival was also worse in patients with higher BVAS: 0.921 (95\%CI $0.775-0.974)$ versus 0.746 (95\%CI 0.619-0.836) ( $p=0.031)$. Early mortality was also predicted by baseline comorbidity score, albumin, CRP and HD requirement on admission in urivariable Cox regression analysis (Table 2). In a multivariable model adjusted for BVAS, age, serum albumin, ANCA type and HD requirement on admission comorbidity score remained a significant predictor for early mortality (HR 1.622, CI 1.006-2.614, $p=0.047$ ) (Table 3).

Late ( $>90$ days after diagnosis) mortality was predicted by age, comorbidity score and HD requirement on admission in univariable analysis (Table 4). In the most fully adjusted model adjusted for age, serum albumin, HD dependency on admission and ANCA type, comorbidity was not a significant predictor any more. In this model, however, BVAS independently predicted all cause late mortality (HR 2.408, 95\%CI 1.081-5.362, Table 5).

Seven patients died of infections (37\%), 4 of cardiovascular diseases (21\%) and 7 of AAV activity (37\%) within the first 90 days. Late mortality occurred from infections in 13 (32\%), cardiovascular diseases in $12(29 \%)$, active AAV during relapse in 6 patients (15\%). Reason for death was unknown in 10 additional cases (1 early, 9 late mortality), and late malignancy was responsible for one death. The following types of infections were documented: bacterial and fungal respiratory tract infections, pulmonary abscess, cerebral abscess, sepsis, disseminated herpes zoster. Both comorbidity and BVAS predicted infectious mortality (HR 2.191, 95\%CI 1.486-3.231; HR 3.792, $95 \%$ CI 1.111-12.949, respectively) in univariable models. The predictive value of comorbidity and BVAS remained significant after adjustment for age, serum albumin, ANCA types, and HD dependency (HR 2.056, 95\%CI 1.247-3.392; HR 5.079, 95\%CI 1.396-18.480, respectively).

By induction immunosuppression remission was achieved in all but one patient, who survived more than 90 days (81 patients, $80 \%$ ). On the long term, ESKD developed in 3 patients who had not required dialysis at diagnosis, but suffered renal failure likely due to low grade persistent disease activity. Thirty-seven patients remained dialysis dependent at study end. Serum creatinine and eGFR in patients who were off dialysis at the end of follow-up $(n=64)$ were $168 \mathrm{umol} / \mathrm{l}(83-434)$ and $33 \mathrm{ml} / \mathrm{min}$ (11-88), respectively. In those, who had BVAS at or above median on admission, serum creatinine at the end of follow-up was significantly higher (191 umol/l (88-418)), compared to patients with BVAS below median (143 umol/l (83-434), $p=0.041)$. The corresponding eGFR values were 26 and $38 \mathrm{ml} / \mathrm{min}$ (11-75 and 12-88, $p=0.092$, respectively). Frequency of long term HD dependency in patients with BVAS at or above and below median did not differ significantly.

Forty relapses occurred in 24 patients, 10 of them experienced 2-4 relapses. The proportion of patients with relapses was $30 \%$ in the BVAS at or above median and $13 \%$ in the BVAS below median group $(p=0.052)$. There was significant difference in the number of relapses between the subgroups with BVAS at or above and below median (34 relapses in 63 patients vs. 6 relapses in 38 patients, $p=0.01$ ). Although BVAS showed association with relapse in univariable logistic regression model ( $\mathrm{OR}=1.130 \mathrm{CI} 1.028-1.243)$, after correcting for the type of ANCA (c-ANCA versus p-ANCA), BVAS was not a significant predictor of relapse any more.

\section{Discussion}

The main result of our analysis is that in AAV patients with predominant renal and pulmonary involvement, comorbidity score independently predicted short term survival. It also proved to be a predictor of infectious mortality. On protocolized immunosuppressive therapy, patients, who had high BVAS at baseline, had significantly poorer short term survival and more frequent relapses than subjects with lower than median score. When analyzing early and late mortality separately, BVAS did not predict outcome in univariable analysis. 
Table 1 Demographics, baseline data and comorbidities at time of diagnosis (mean (SD) or median and range)

\begin{tabular}{|c|c|c|c|c|}
\hline Variable & All patients & $\begin{array}{l}\text { Patients } \\
\text { with BVAS } \geq 21\end{array}$ & $\begin{array}{l}\text { Patients } \\
\text { with BVAS < } 21\end{array}$ & $p$ value \\
\hline$n$ of patients & 101 & 63 & 38 & \\
\hline Age (years) & $61.4(13)$ & $60.2(14)$ & $63.3(11)$ & 0.237 \\
\hline Male/female & $40 / 61$ & $27 / 36$ & $13 / 25$ & 0.389 \\
\hline Time from first symptoms to diagnosis (months) & $5.0(1-36)$ & $5.0(1-24)$ & $6.0(1-36)$ & 0.822 \\
\hline Hemoglobin (g\%) & $8.4(1.4)$ & $8.1(1.3)$ & $8.8(1.5)$ & 0.017 \\
\hline Erythrocyte sedimentation rate (mm/h) & $98(5-138)$ & $98(5-138)$ & $99(14-136)$ & 0.762 \\
\hline Serum albumin $(g / l)$ & $31.3(5.4)$ & $30.8(5.3)$ & $32.0(5.6)$ & 0.285 \\
\hline CRP (mg/l) & $29(1-221)$ & $40(2-221)$ & $24(1-152)$ & 0.054 \\
\hline Urinary protein excretion (mg/day) & $1456(38-8474)$ & $1259(38-8474)$ & $1843(184-7344)$ & 0.143 \\
\hline Serum creatinine (umol/l) & $554(84-1904)$ & $573(146-1904)$ & $428(84-1722)$ & 0.060 \\
\hline HD requirement on admission $(n, \%)$ & $56(55 \%)$ & $41(65 \%)$ & $15(40 \%)$ & 0.012 \\
\hline BVAS & $21(11-34)$ & $24(21-34)$ & $15(11-20)$ & $<0.001$ \\
\hline p-/c-ANCA positivity $(n)^{*}$ & $57 / 36$ & $33 / 29$ & $24 / 7$ & 0.024 \\
\hline Anti-MPO level in p-ANCA positive patients (IU/ml) & $67(6-100)$ & $67(6-100)$ & $70(11-100)$ & 0.807 \\
\hline Anti-PR3 level in c-ANCA positive patients (IU/ml) & $100(32-100)$ & $100(50-100)$ & $82(32-100)$ & 0.354 \\
\hline Dose of iv pulse MP** mg/kg/bw & $11.7(4.1)$ & $12.7(4.2)$ & $10.1(3.3)$ & 0.002 \\
\hline Dose of iv bolus CYC ${ }^{* * *} \mathrm{mg} / \mathrm{kg} / \mathrm{bw}$ & $9.7(1.6)$ & $9.8(1.6)$ & $9.5(1.6)$ & 0.371 \\
\hline Cumulative dose of MP (mg) & $11640(3006-32334)$ & $11238(3006-32334)$ & $12332(5076-26364)$ & 0.621 \\
\hline Follow-up time (days) & $963(30-3000)$ & $843(30-3000)$ & $1393(51-3000)$ & 0.231 \\
\hline \multicolumn{5}{|l|}{ Organ involvement n (\%) } \\
\hline Renal & $101(100)$ & $63(100)$ & $38(100)$ & 1.000 \\
\hline Respiratory tract & $43(43)$ & $38(60)$ & $5(13)$ & $<0.001$ \\
\hline Ear-throat-nose & $39(39)$ & $33(52)$ & $6(16)$ & $<0.001$ \\
\hline Musculoskeletal & $55(55)$ & $33(52)$ & $22(58)$ & 0.590 \\
\hline Skin & $16(16)$ & $9(14)$ & $7(18)$ & 0.581 \\
\hline Eyes & $5(5)$ & $4(6)$ & $1(3)$ & 0.648 \\
\hline Gastrointestinal & $9(9)$ & $9(14)$ & $0(0)$ & 0.013 \\
\hline Nervous system & $17(17)$ & $14(22)$ & $3(8)$ & 0.062 \\
\hline Cardiovascular & $5(5)$ & $5(8)$ & $0(0)$ & 0.154 \\
\hline Baseline comorbidities n (\%) & $963(30-3000)$ & $843(30-3000)$ & $1393(51-3000)$ & 0.231 \\
\hline \multicolumn{5}{|l|}{ History of: } \\
\hline Coronary artery disease & $11(11)$ & $9(14)$ & $2(5)$ & 0.201 \\
\hline Congestive heart failure & $5(5)$ & $4(6)$ & $1(3)$ & 0.648 \\
\hline Peripheral vascular dis. & $1(1)$ & $1(2)$ & $0(0)$ & 1.000 \\
\hline Cerebrovascular disease & $11(11)$ & $7(11)$ & $4(11)$ & 1.000 \\
\hline Chronic pulmonary dis. & $16(16)$ & $9(14)$ & $7(18)$ & 0.581 \\
\hline Peptic ulcer disease & $8(8)$ & $6(10)$ & $2(5)$ & 0.707 \\
\hline Liver disease & $5(5)$ & $4(6)$ & $1(3)$ & 0.648 \\
\hline Diabetes mellitus & $8(8)$ & $3(5)$ & $5(13)$ & 0.149 \\
\hline Malignancy & $7(7)$ & $3(5)$ & $4(11)$ & 0.421 \\
\hline \multicolumn{5}{|l|}{ Number of patients with } \\
\hline 0 & 50 & 30 & 20 & 0.862 \\
\hline 1 & 30 & 19 & 11 & \\
\hline 2 or more comorbidity scores & 21 & 14 & 7 & \\
\hline
\end{tabular}

*Eight patients were ANCA negative, all of them had renal biopsy which proved the diagnosis of pauci-immune crescentic glomerulonephritis ${ }^{* *} \mathrm{MP}$ - methylprednisolone, administered for 98 patients

${ }^{* * *}$ CYC - cyclophosphamide, administered for 95 patients

Comorbidity scores were given 0 if no comorbidity, 1 if a single comorbidity, 2 if two or more comorbidities existed 


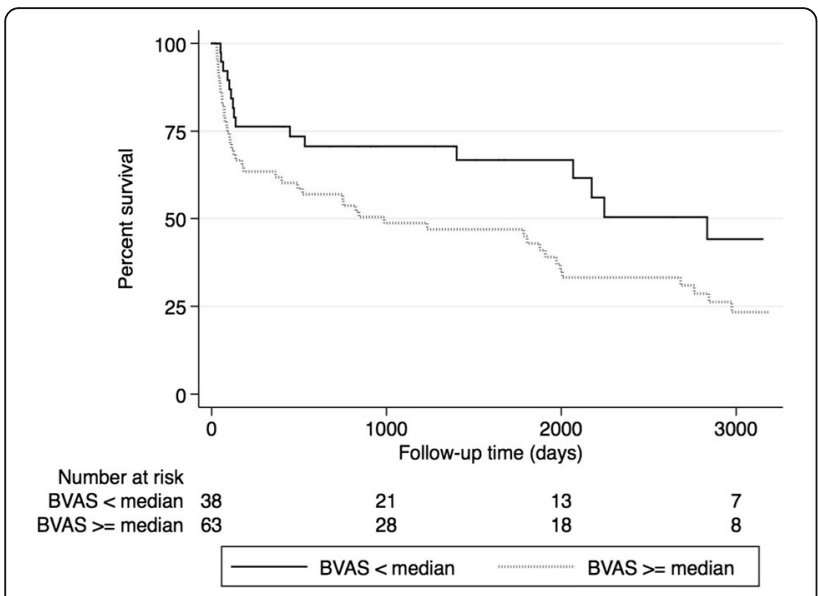

Fig. 1 Kaplan-Meier survival curves of patients with BVAS at or above and below median in the entire observation period $(p=0.028$, log-rank test)

BVAS, originally designed to standardize disease assessment in AAV, shows good correlation with clinical activity of the disease [8]. Flossmann et al. documented, that BVAS was a significant predictor of mortality by analyzing the data of patients recruited for randomized controlled trials. Patients in that study were somewhat different from the ones enrolled in ours, since the median BVAS was lower, renal function was less severely compromised, and subjects with life-threatening pulmonary hemorrhage were excluded [1]. On the contrary, predictive value of BVAS was not found in several other investigations. Bakoush and coworkers followed 83 patients; neither survival nor ESKD was predicted by BVAS in their cohort, with less severe renal failure compared to our patients [9]. In Japanese patients with MPOANCA disease, no association was found between BVAS and mortality during the two years follow-up [20]. In another investigation there was no difference between the

Table 2 Predictors of "early mortality" in univariable Cox regression analysis

\begin{tabular}{llll}
\hline & HR & $95 \%$ Cl & $P$ value \\
\hline Age & 1.035 & $0.994-1.078$ & 0.097 \\
Albumin & 0.891 & $0.818-0.970$ & 0.007 \\
ANCA type - ANCA neg & REF & REF & REF \\
PANCA & 0.604 & $0.215-1.695$ & 0.338 \\
CANCA & 0.516 & $0.067-3.945$ & 0.524 \\
BVAS median & 3.57 & $1.039-12.243$ & 0.043 \\
CRP & 1.011 & $1.003-1.018$ & 0.005 \\
Comorbidity score & 1.707 & $1.176-2.477$ & 0.005 \\
HD on admission & 3.404 & $1.130-20.260$ & 0.030 \\
\hline
\end{tabular}

Table 3 Comorbidity score predicts "early mortality" in multivariable Cox regression analysis. Table shows the parameters of the "comorbidity score" variable in different models

\begin{tabular}{llll}
\hline & HR & $95 \% \mathrm{Cl}$ & $P$ value \\
\hline Model 1 & 1.707 & $1.176-2.477$ & 0.005 \\
Model 2 & 1.752 & $1.225-2.506$ & 0.002 \\
Model 3 & 1.694 & $1.072-2.677$ & 0.024 \\
Model 4 & 1.622 & $1.006-2.614$ & 0.047 \\
\hline
\end{tabular}

Model 1: comorbidity score

Model 2: Model $1+$ BVAS median

Model 3: Model 2+ Age, serum albumin

Model 4: Model $3+\mathrm{HD}$ dependency on admission and ANCA type (c-versus p-ANCA)

baseline BVAS of survivors and non-survivors; baseline BVAS did not, but BVAS at 1 and 3 months predicted survival [21].

The difference in the association between BVAS and outcome in these cohorts and ours can be due to a variety of factors. Event number, therefore statistical power, patient selection, disease severity and treatment approach were quite heterogeneous across these studies. It also seems important to differentiate early and late survival, as the hazard of mortality is not proportional in these periods. We have defined the timeframe of early death in 3 months, as risk of severe complications of AAV, also intensity of immunosuppression are the highest during this period.

To our knowledge, only one study has investigated the association between comorbidities and risk of all cause death in AAV patients. Little et al. found, that the Karnofsky performance score, but not the nonvasculitic comorbidity showed independent association with mortality [22]. Although we did not include Karnofsky performance in our dataset, we found a significant association of comorbidity and early mortality, and this relationship remained independent of other important clinical characteristics.

Table 4 Predictors of "late mortality" in univariable Cox regression analysis

\begin{tabular}{llll}
\hline & HR & $95 \% \mathrm{Cl}$ & $P$ value \\
\hline Age & 1.059 & $1.028-1.092$ & $<0.001$ \\
Albumin & 0.950 & $0.895-1.009$ & 0.094 \\
ANCA type - ANCA neg & REF & REF & REF \\
pANCA & 0.658 & $0.341-1.268$ & 0.211 \\
CANCA & 0.674 & $0.159-2.853$ & 0.592 \\
BVAS median & 1.483 & $0.768-2.865$ & 0.241 \\
CRP & 0.999 & $0.993-1.007$ & 0.993 \\
Comorbidity score & 1.526 & $1.106-2.106$ & 0.010 \\
HD on admission & 2.157 & $1.131-4.116$ & 0.020 \\
\hline
\end{tabular}


Table 5 BVAS predicts "late mortality" in multivariable Cox regression analysis. The table shows the parameters of the "BVAS median" variable in the different models

\begin{tabular}{lllc}
\hline & HR & $95 \% \mathrm{Cl}$ & $P$ value \\
\hline Model 1 & 1.483 & $0.768-2.865$ & 0.241 \\
Model 2 & 2.073 & $1.030-2.435$ & 0.041 \\
Model 3 & 2.558 & $1.251-5.231$ & 0.010 \\
Model 4 & 2.408 & $1.081-5.362$ & 0.031 \\
\hline
\end{tabular}

Model 1: BVAS median

Model 2: Model $1+$ comorbidity score

Model 3: Model 2+ Age, serum albumin

Model 4: Model 3+ HD dependency on admission and ANCA type (cversus $\mathrm{p}-\mathrm{ANCA}$ )

We did not find other investigations assessing the association between comorbidities and infectious death. Importantly, this reveals the complexity of treatment of AAV patients: likely those without any comorbidity may tolerate aggressive immunosuppression and AAV better, compared to subjects suffering from various chronic disorders. Remarkably, in another study, accumulation of adverse events in the first year of treatment - which influenced survival significantly - was independently associated with age and renal impairment [2]. Based on these latter findings we propose, that not only age and kidney function, but also the presence of non-vasculitic comorbidities present high risk status for adverse events, especially for infections, which may provide an explanation for the increased mortality.

Our findings are in accord with several other reports showing that the severity of kidney disease at baseline is an indicator of poor prognosis $[1,6,9,10]$. Similarly, high levels of the inflammatory markers (CRP, albumin, etc.) confer an increased early mortality risk for the individual patient [5, 7]. The applicability of these predictors is important, as these are readily available at the first presentation of the patient.

The frequency of ESKD did not differ in the BVAS groups in our cohort, similarly to other investigations $[4,23]$. We found more frequent relapses in patients with higher BVAS; BVAS predicted relapse in Cox regression analysis, but the association was not significant after adjustment for ANCA type. The likely explanation for this observation is, that the higher BVAS in our cohort, comprising patients with both respiratory tract and kidney involvement, was associated with c-ANCA disease, which characteristically confers a higher risk of relapse compared to $p$-ANCA positive vasculitis [24]. In comparison, in a study investigating patients exclusively with c-ANCA positivity, relapses occurred more often in those who presented with lower BVAS, compared to more severe cases [25]. A possible explanation for this difference can be the different case mix: cohorts with predominantly upper respiratory tract involvement but no kidney disease have lower BVAS but more relapses than those with renal AAV [26].

Our study has several limitations. Most importantly, BVAS was calculated retrospectively. Nevertheless, detailed source data provided reliable information, and the strictly followed treatment protocol also assisted our analysis. Mortality rate was fairly high, which likely can be explained by very late referrals. This resulted in advanced renal failure and extensive manifestations of AAV in most of our patients. The extensive comorbidities might also have contributed to the observed high mortality.

In conclusion, baseline comorbidities influence both short and long term outcome of patients with AAV. Risk-stratification would help clinicians to tailor therapy individually, which might further improve the outcome. Future prospective treatment studies are needed to assess whether scoring systems based on comorbidities and BVAS help to individualize therapies in order to improve short and long term survival.

\section{Abbreviations \\ AAV: ANCA-associated vasculitis; BVAS: Birmingham Vasculitis Activity Score; Cl: Confidence interval; CYC: Cyclophosphamide; eGFR: Estimated GFR; ESKD: End stage kidney disease; HR: Hazard ratio; iv: Intravenous; MP: Methylprednisolone; OR: Odds ratio}

\section{Acknowledgements}

Authors are indebted to Dr. Andras Keszei (clinical epidemiologist, Department of Medical Informatics, RWTH Aachen University) for his statistical advice in revising the manuscript.

\section{Funding}

Not applicable.

Availability of data and materials

Anonymised data are available upon request to authors.

\section{Authors' contributions}

Each author (ÁH, KP, JA, HB, IK, LR, GK and IM) contributed to the design of this investigation, the analysis of the data and the preparation of the manuscript. All authors read and approved the final manuscript.

\section{Competing interest}

The authors declare that they have no competing interests.

\section{Consent to publish}

Not applicable.

\section{Ethics approval and consent to participate}

The study was approved by the Institutional Ethics Committee of Szent Margit Hospital, Budapest, Hungary, and conducted in agreement with the declaration of Helsinki. Since this was a retrospective analysis of patient records, a waiver for consent has been obtained from the institutional ethics board.

\section{Author details}

${ }^{1}$ Nephrology Department, Szent Margit Hospital, 132 Bécsi út, Budapest 1032 Hungary. ${ }^{2}$ Pathology Department, Szent Margit Hospital, 132 Bécsi út, Budapest 1032, Hungary. ${ }^{3}$ Institute of Pathophysiology, Semmelweis University, 4 Nagyvárad tér, Budapest 1089, Hungary. ${ }^{4}$ Department of Medicine (Nephrology), University of Toronto, Kidney Transplant Program, Toronto General Hospital, University Health Network, 585 University Avenue, Toronto M5G 2 N2, ON, Canada. 
Received: 2 December 2015 Accepted: 15 February 2017

Published online: 23 February 2017

\section{References}

1. Flossmann O, Berden A, de Groot K, Hagen C, Harper L, Heijl C, et al. Long-term patient survival in ANCA-associated vasculitis. Ann Rheum Dis. 2011;70:488-94.

2. Little MA, Nightingale P, Verburgh CA, Hauser T, De Groot K, Savage C, et al. Early mortality in systemic vasculitis: relative contribution of adverse events and active vasculitis. Ann Rheum Dis. 2010;69:1036-43.

3. Satchell SC, Nicholls AJ, D'Souza RJ, Beaman M. Renal vasculitis: Increasingly a disease of the elderly? Nephron Clin Pract. 2004;97:c142-146.

4. Corral-Gudino L, Borao-Cengotita-Bengoa M, del Pino-Montes J, LermaMarquez JL. Overall survival, renal survival and relapse in patients with microscopic polyangiitis: a systematic review of current evidence. Rheumatology. 2011;50:1414-23.

5. Briedigkeit L, Kettritz R, Göbel U, Natusch R. Prognostic factors in Wegener's granulomatosis. Postgrad Med J. 1993;69:856-61.

6. Slot MC, Tervaert JWC, Franssen CFM, Stegeman CA. Renal survival and prognostic factors in patients with PR3-ANCA associated vasculitis with renal involvement. Kidney Int. 2003;63:670-7.

7. Yamagata K, Usui J, Saito C, Yamaguchi N, Hirayama K, Mase K, et al. ANCA-associated systemic vasculitis in Japan: clinical features and prognostic changes. Clin Exp Nephrol. 2012;16:580-8.

8. Mukhtyar C, Lee R, Brown D, Carruthers D, Dasgupta B, Dubey S, et al. Modification and validation of the Birmingham Vasculitis Activity Score (version 3). Ann Rheum Dis. 2009;68:1827-32.

9. Bakoush O, Segelmark M, Torffvit O, Ohlsson S, Tencer J. Urine IgM excretion predicts outcome in ANCA-associated renal vasculitis. Nephrol Dial Transplant. 2006;21:1263-9.

10. Bourgarit A, Toumelin PL, Pagnoux C, Cohen P, Mahr A, Guern VL, et al. Deaths occurring during the first year after treatment onset for polyarteritis nodosa, microscopic polyangiitis, and Churg-Strauss syndrome. Medicine. 2005;84:323-30.

11. Frausova D, Brejnikova M, Hruskova Z, Rihova Z, Tesar V. Outcome of thirty patients with ANCA-associated renal vasculitis admitted to the intensive care unit. Ren Fail. 2008;30:890-5.

12. Ahn JK, Hwang JW, Lee J, Jeon $\mathrm{CH}$, Cha HS, Koh EM. Clinical features and outcome of microscopic polyangiitis under a new consensus algorithm of ANCA-associated vasculitis in Korea. Rheumatol Int. 2012;32:2979-86.

13. Watanabe K, Tani Y, Kimura H, Tanaka K, Hayashi Y, Asahi K, et al. Clinical outcomes of Japanese MPO-ANCA-related nephritis: Significance of initial renal death for survival. Intern Med. 2012;51:1969-76.

14. Hemmelgarn BR, Manns BJ, Quan H, Ghali WA. Adapting the Charlson Comorbidity Index for use in patients with ESRD. Am J Kidney Dis. 2003;42:12532.

15. Rattanasompattikul M, Feroze U, Molnar MZ, Dukkipati R. Kovesdy CsP, Nissenson AR, et al. Charlson comorbidity score is a strong predictor of mortality in hemodialysis patients. Int Urol Nephrol. 2012;44:1813-23.

16. Jennette JC, Falk RJ, Andrassy K, Bacon PA, Churg J, Gross WL, et al. Nomenclature of systemic vasculitides. Proposal of an international consensus conference. Arthritis Rheum. 1994;37:187-92

17. Jennette JC, Falk RJ, Bacon PA, Basu N, Cid MC, Ferrario F, et al. 2012 Revised International Chapel Hill Consensus Conference Nomenclature of Vasculitides. Arthritis Rheum. 2013:65:1-11.

18. Levey AS, Stevens LA, Schmid CH, Zhang YL, Castro AF, Feldman Hl, et al. A new equation to estimate glomerular filtration rate. Ann Intern Med. 2009; 150:604-12.

19. Vittinghoff $E, M c C u l l o c h ~ C E$. Relaxing the rule of ten events per variable in logistic and Cox regression. Am J Epidemiol. 2007;165:710-8.

20. Koike K, Fukami K, Yonemoto K, Iwatani R, Obata R, Ueda K, et al. A new vasculitis activity score for predicting death in myeloperoxidase-antineutrophil cytoplasmic antibody-associated vasculitis patients. Am J Nephrol. 2012;35:1-6.

21. Itabashi M, Takei T, Yabuki Y, Suzuki H, Ando M, Akamatsu M, et al. Clinical outcome and prognosis of anti-neutrophil cytoplasmic antibody-associated vasculitis in Japan. Nephron Clin Pract. 2010;115:c21-27.

22. Little MA, Nazar L, Farrington K. Outcome in glomerulonephritis due to systemic small vessel vasculitis: effect of functional status and non-vasculitic co-morbidity. Nephrol Dial Transplant. 2004;19:356-64.

23. Kawai H, Banno S, Kikuchi S, Nishimura N, Nobata H, Kimura Y, et al. Retrospective analysis of factors predicting end-stage renal failure or death in patients with microscopic polyangiitis with mainly renal involvement. Clin Exp Nephrol. Doi: 10.1007/s10157-013-0926-1.

24. Lionaki S, Blyth ER, Hogan SL, Hu Y, Senior JBA, Jennette CE, et al. Classification of ANCA vasculitides: The role of anti-neutrophil cytoplasmic autoantibody specificity for MPO or PR-3 in disease recognition and prognosis. Arthritis Rheum. 2012;64:3452-62.

25. Yegin EG, Can M, Yilmaz N, Aydin SZ, Yavuz S, et al. Activity and damage in granulomatosis with polyangiitis. Int J Rheum Dis. 2013;16:61-71.

26. Walsh M, Flossmann O, Berden A, Westman K, Höglund P, Stegeman C, Jayne D. Risk factors for relapse of antineutrophil cytoplasmic antibody-associated vasculitis. Arthritis Rheum. 2012:64:542-8.

\section{Submit your next manuscript to BioMed Central and we will help you at every step:}

- We accept pre-submission inquiries

- Our selector tool helps you to find the most relevant journal

- We provide round the clock customer support

- Convenient online submission

- Thorough peer review

- Inclusion in PubMed and all major indexing services

- Maximum visibility for your research

Submit your manuscript at www.biomedcentral.com/submit
C) Biomed Central 